\title{
Astrophysical consequences of the screening of nuclear reactions
}

\section{J. ISERN AND M.HERNANZ}

Centre d'Estudis Avançats Blanes (CSIC), Cami de Santa Bàrbara sn, 17900

Blanes, Spain.

\begin{abstract}
The rate of nuclear reactions depends on the influence of the surrounding particles that compose the plasma. At high densities the situation is far from being satisfactory and the influence of electron polarization has not been completely elucidated. In particular, it is shown that the possibility of an accretion induced collapse of a carbon-oxygen white dwarf instead of a supernova explosion completely depends on the screening factors and pycnonuclear rates that are adopted. Similarly, the possibility of detecting isolated neutron stars that accrete matter from the interstellar medium depends on the adopted pycnonuclear rates. Low rates allow the formation of a metastable layer that can release energy explosively and produce a $\gamma$-ray burst. Nevertheless, current rates seem to prevent such a situation.

Le taux des réactions nucléaires dépend de l'influence exercée par les particules voisines qui composent le plasma. A haute densité, la situation est loin d'être satisfaisante et l'influence de la polarization electronique n'est pas sufisamment claire. En particulier, on montre que la possibilité d'obtenir un collapse non explosif d'une naine blanche de carbone oxygène dépend des facteurs d'écrantage et des taux pycnonucléaires adoptés. Egalement, la possibilité de détecter des étoiles à neutrons isolées dépend des taux pycnonucléaires adoptés. Des petites valeurs favorisent la formation d'une couche metastable qui peut libérer de l'energie explosivement et produire
\end{abstract}


une éruption gamma. Quand- même, les taux actuels semblent empêcher cette situation

\subsection{Introduction}

A nuclear reaction happens when two nuclei approach to a distance of the order of $10^{-13} \mathrm{~cm}$ after tunneling the Coulomb barrier. In the case of two isolated nuclei, this barrier is described by:

$$
V_{12}(r)=\frac{Z_{1} Z_{2} e^{2}}{r}
$$

but in the case of a plasma it must be modified to account for the influence of the environment. It is possible to write:

$$
V_{12}(r)=\frac{Z_{1} Z_{2} e^{2}}{r}+w(r)
$$

where $w(r)$ represents the average contribution of the surrounding particles to the interaction potential. Since the effect of this term is to lower the barrier, the transmission coefficient increases and the reaction rate can drastically differ from the case of isolated nuclei. For instance, in the case of a pure carbon plasma with a density $\rho=10^{9} \mathrm{~g} / \mathrm{cm}^{3}$ and a temperature $T=10^{9} \mathrm{~K}$, the rate of the reaction ${ }^{12} \mathrm{C}+{ }^{12} \mathrm{C}$ increases by a factor of $10^{16}$ due to the influence of the surrounding particles. This effect was noticed for the first time by Schatzman (1948) and was later developed by several authors (Salpeter, 1954; Salpeter and Van Horn, 1969; DeWitt et al, 1973; Graboske et al, 1973; Jancovici, 1977; Alastuey and Jancovici, 1978; Itoh et al, 1978, 1979, 1990; Yakovlev and Shalybkov, 1988; Ogata et al 1991).

Usually, the screening factor is written as

$$
E=\frac{R}{R_{0}}
$$

where $\mathbf{R}$ and $\mathbf{R}_{0}$ are the rate of thermonuclear reactions with and without allowance for screening. If $|E-1|<<1$, the screening is called weak. If $|E-1|>>1$, it is called strong. In the first case, the theory developed by DeWitt et al (1973), Graboske et al (1973) or Yakovlev and Shalybkov (1988) is completely satisfactory from the astrophysical point of view since corrections are usually smaller than the current uncertainties in the astrophysical factors. This regime almost covers the evolution of stars in the main sequence (except the low luminosity end) but not the advanced stages of the evolution of intermediate stars.

Once $\mathrm{H}$ is exhausted in the central regions, intermediate and low mass 
stars develop an electron degenerate core. The typical densities of these cores do not allow to neglect the screening effects on nuclear reactions, which turn out to be dominant. This regime is not yet completely understood (see Rosenfeld, this volume), specially at high densities and improvements can change the chemical composition of degenerate cores or even the loci of the ignition curve in the cold, high density regions of the $\rho$-T plane.

If densities are high enough, the barrier penetration is driven by the energy of the ground state of nuclei in a lattice instead of by the thermal energy and nuclear fusion happens in the so called pycnonuclear regime. This implies that, even at zero temperature, nuclear fusion can happen and that it is not possible to reach arbitrarily high densities without a nuclear rearrangement of matter. The transition from the strong screening to the pycnonuclear regime is not well known and all the work done up to now relies on interpolations that try to avoid discontinuities.

A correct understanding of both regions, pycnonuclear and strong screening, is crucial in order to elucidate if $\mathrm{CO}$ white dwarfs can make a silent collapse to a neutron star or they will always explode as a Type Ia supernova when they accrete enough matter from a companion star. Both regions, specially the pycnonuclear one, are also relevant to elucidate if the long term evolution of slowly accreting neutron stars can result into a $\gamma$-ray burst, a thermonuclear explosion or just into a peaceful growth of their mass. Here in this paper, we will only address to the fate of accreting $\mathrm{CO}$ white dwarfs and accreting neutron stars.

\subsection{The physical problem}

Dense plasmas can be characterized by several dimensionless parameters which facilitate the classification of physical properties in each problem. In order to simplify, it is possible to consider a plasma consisting of only one chemical species with atomic and mass numbers $\mathrm{Z}$ and $\mathrm{A}$ respectively, with a ion number density $n_{i}$ (or, quivalently, a density $\rho=A H n_{i}$, where $\mathrm{H}$ is the atomic mass number) and a temperature $T$.

The ion system can be described through the following parameters: The strength of the Coulomb coupling of ions is described by the so called Coulomb coupling constant, $\Gamma$,

$$
\Gamma=\frac{Z^{2} e^{2}}{a k T}
$$

where $\mathrm{a}$ is the ion-sphere or Wigner-Seitz radius, $a=\left(3 / 4 \pi n_{i}\right)^{1 / 3}$. A weakly coupled plasma satisfies the relationship $\Gamma<<1$, while a strongly coupled 
plasma satisfies $\Gamma \geq 1$. When $\Gamma \geq 180$, the ion plasma suffers a phase transition and solidifies into a bcc crystal (Slattery, Doolen, DeWitt, 1980, 1982; Helfer, McCrory, Van Horn 1984; Ogata and Ichimaru, 1987)

The ratio between the thermal de Broglie wavelength of an ion, $\lambda_{T}$, and the ionic spacing, a, describes the importance of the quantum effects in the description of the fluid properties.

$$
\Lambda=\frac{\lambda_{T}}{a}=\frac{\hbar \sqrt{2 \pi}}{a \sqrt{2 m k T}}
$$

A classical fluid satisfies $\Lambda<<1$.

The importance of the quantum effects can also be outlined through the parameter $\eta$ as:

$$
\eta=\frac{\hbar \omega_{p}}{k T}
$$

where $\omega_{p}$ is the ionic plasma frequency.

$$
\omega_{p}=\frac{Z e}{A H} \sqrt{4 \pi \rho}=Z e \sqrt{\frac{4 \pi n_{i}}{3 A H}}
$$

Values of $\eta>1$ imply that the contribution of the quantum plasma effects to the reaction rates cannot be longer neglected.

The electron component can be characterized through its Wigner-Seitz radius, usually expressed in units of the Bohr radius (Pines and Nozières, 1966):

$$
r_{s}=\frac{a_{e}}{a_{B}}
$$

where $a_{e}=\left(3 / 4 \pi n_{e}\right)^{1 / 3}$ and $a_{B}=\hbar^{2} / m e^{2}=5.29210^{-9}$. The Fermi momentum of electrons in units of mc is given by

$$
x_{F}=\frac{\hbar\left(3 \pi^{2} n_{e}\right)^{1 / 3}}{m c}=\frac{1.4010^{-2}}{r_{s}}
$$

while the Fermi energy, in units of $\mathrm{mc}^{2}$, is given by

$$
E_{F}=m c^{2}\left(\sqrt{1+1.9610^{-4} r_{s}^{-2}}-1\right)
$$

The degree of degeneracy can be expressed through the well known parameter

$$
\Psi=\frac{\mu}{k T} \simeq \frac{E_{F}}{k T}
$$

where $\mu$ is the chemical potential of electrons. 
The coupling strength between electrons and nuclei is given by the rate between the Thomas-Fermi screening length and the radius of the electronic sphere $\mathrm{a}_{e}$

$$
\frac{\lambda_{T F}}{a_{e}}=\left(\frac{\pi}{12}\right)^{1 / 3}\left(\frac{a_{e}}{a_{B}}\right)^{-1 / 2}
$$

For densities higher than $10^{6} \mathrm{~g} / \mathrm{cm}^{3}$, the electrons are weakly polarized and they can be treated as an ideal Fermi gas $\left(\lambda_{T F} / a_{e}>>1\right)$.

The temperature is usually referred to the Gamow energy, $\mathrm{F}_{G}$. In the case of nuclei interacting via the bare Coulomb potential (case of a tenuous plasma), the rate, $R_{0}$, of the reaction is

$$
R_{0} \propto \exp \left(-3 E_{G} / k T\right)
$$

and the parameter $\tau$, that only depends on the temperature, is defined as

$$
\tau=\frac{3 E_{G}}{k T}=\left[\left(\frac{27 \pi^{2}}{4}\right) \frac{2 \mu Z_{1}^{2} Z_{2}^{2} e^{4}}{k T \hbar^{2}}\right]^{1 / 3}
$$

where $\mu$ is the reduced mass of the colliding particles. The radius, $r_{0}$, of the classical turning point for colliding pairs with an energy equal to the Gamow energy is (Alastuey and Jancovici 1978)

$$
\frac{r_{0}}{a}=\frac{3 \Gamma}{\tau}=\frac{2}{\pi}^{2 / 3} \eta^{2 / 3}
$$

\subsubsection{Strong screening regime}

\subsubsection{Nonresonant reactions}

In the case of nonresonant reactions, the reaction rate can be written as:

$$
R \propto \int_{0}^{\infty} S(E) P(E) \exp \left(-\frac{E}{k T}\right) d E
$$

where $P(E)$ is the Coulomb barrier-penetration factor, the exponential term is the Boltzmann factor and $S(E)$ is the astrophysical factor. Since $S(E)$ is a smooth function of the energy and $P(E) \exp (-E / k T)$ displays a pronounced peak around the Gamow energy, $\mathrm{E}_{G}$, it is possible to write:

$$
R \propto S\left(E_{G}\right) \int_{0}^{\infty} P(E) \exp \left(-\frac{E}{k T}\right) d E
$$

and the integral can be interpreted as the pair correlation function at a distance of the order of the nuclear radius, $g\left(r_{n}\right)$ (DeWitt, Graboske and 
Cooper, 1973; Graboske et al, 1973; Alastuey and Jancovici, 1978; Yakovlev and Shalybkov, 1988):

$$
g(r) \propto \exp \left|-\frac{V_{12}(r)}{k T}\right|
$$

Therefore, the enhancement factor can be written as

$$
E=\frac{S\left(E_{G}\right)}{S\left(E_{G_{0}}\right)} \frac{g\left(r_{n}\right)}{g_{0}\left(r_{n}\right)}
$$

where $E_{G_{0}}$ and $g_{0}$ are the Gamow energy and the pair correlation function for nuclei in an infinitely diluted plasma. Despite $g\left(r_{n}\right)$ is a strongly varying function in the neighborhood of $r_{n}=0$, the ratio $g\left(r_{n}\right) / g_{0}\left(r_{n}\right)$ is a slowly varying function of $r_{n}$ and it is possible to write

$$
R \approx \frac{g(0)}{g_{0}(0)}
$$

It has been shown (Jancovici, 1977; Yakovlev and Shalybkov, 1988) that

$$
E=\exp \left(-\frac{\Delta F}{k T}-\phi(\Gamma, \eta)\right)
$$

where the first term, which is dominant, corresponds to the purely classical contribution and is equal to the difference between the Coulomb excess of free energies after and before the interaction, and the second term contains the quantum corrections. This term, which is not negligible at all at high densities (Figure 1), has been computed by several authors using different methods: the WKB approximation (Itoh et al, 1977, 1979), the path integral (Alastuey and Jancovici, 1978) or directly (Ogata et al, 1991). A critical discussion of the consistency of the approximations involved in these calculations can be found in Yakovlev and Shalybkov (1989) and in Rosenfeld (this volume). Figure 2 displays the values of $\ln E$ as a function of $\Gamma$ for two values of $3 \Gamma / \tau$, and in section 3 we discuss the astrophysical consequences of using different approximations.

\subsubsection{Resonant reactions}

In the case of resonant reactions, their rate can be written as:

$$
R \propto \frac{\Gamma_{\text {in }}\left(E_{r}\right) \Gamma_{o u t}\left(E_{r}\right)}{\Gamma_{\text {tot }}\left(E_{r}\right)} \exp \left(-\frac{E_{r}}{k T}\right)
$$

where $\mathrm{E}_{r}$ is the resonant energy and $\Gamma_{\text {in }}, \Gamma_{\text {out }}$ are the width of the direct and reverse reactions respectively, and $\Gamma_{\text {tot }}=\Gamma_{\text {in }}+\Gamma_{\text {out }}$ is the total width. In the case of a strongly coupled plasma, the resonant energy is shifted to $E_{r}=$ 


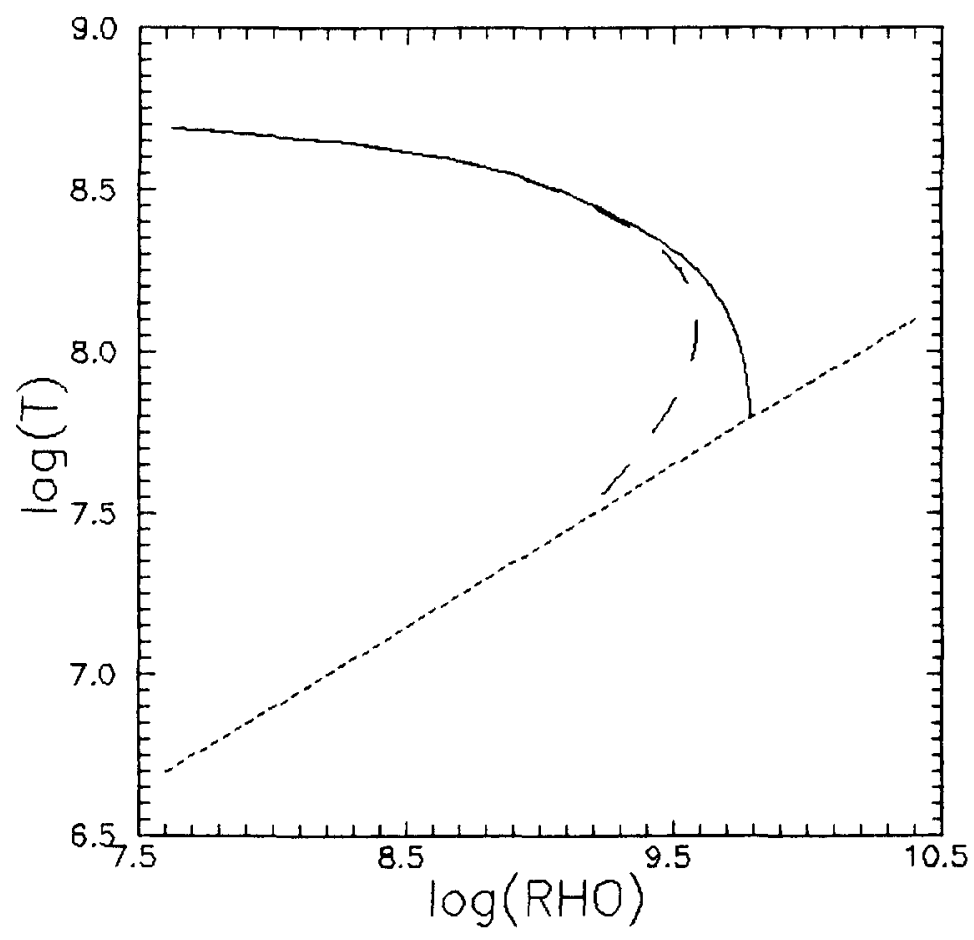

Fig. 5.1 Ignition line for a pure carbon plasma. The continuous line has been computed using the full expression of Alastuey and Jancovici (1978). The dashed line corresponds to the case where only the purely classical contribution is used. The dotted line, $3 \Gamma / \tau=1.6$, outlines the limit of validity of the expression

$E_{r}+\Delta F$ and the enhancement factor becomes (Mitler 1977, Mochkovitch and Nomoto, 1986):

$$
E=\frac{\Gamma_{i n}\left(E_{r}^{\prime}\right) \Gamma\left(E_{r}\right)}{\Gamma_{i n}\left(E_{r}\right) \Gamma\left(E_{r}^{\prime}\right)} \exp \left(-\frac{\Delta F}{k T}\right)
$$

\subsubsection{Photodesintegration reactions}

The photodesintegration rate of a nucleus is given by

$$
\lambda \propto<\sigma v>\exp \left(-Q_{0} / k T\right)
$$

where $\langle\sigma v\rangle$ is the rate per pair of interacting particles of the forward reaction:

$$
<\sigma v>\propto \int_{0}^{\infty} \sigma(E) \exp (-E / k T) d E
$$




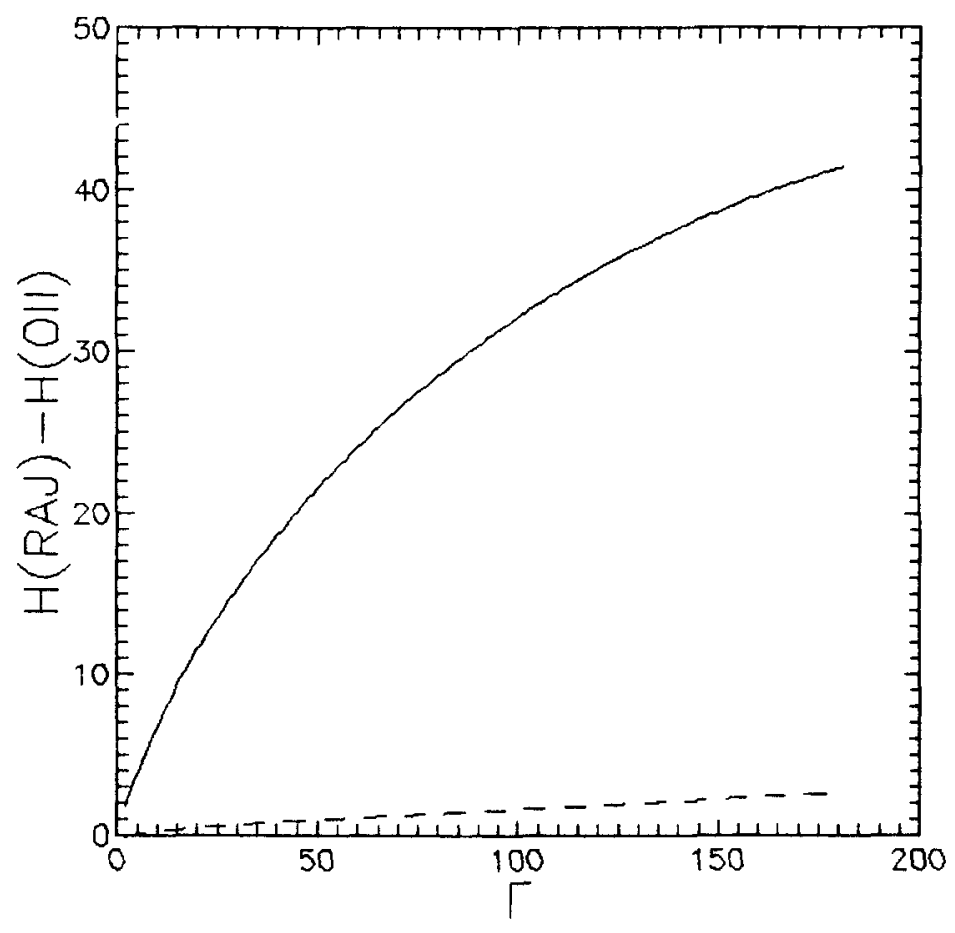

Fig. 5.2 Comparison of the screening factors obtained by Alastuey and Jancovici (1978), updated with the values proposed by Rosenfeld (this volume), H(RAIJ), and those obtained by Ogata et al (1991), H(OII). The continuous line corresponds to $3 \Gamma / \tau=1$ and the dashed line to $3 \Gamma / \tau=0.5$

$E$ is the kinetic energy in the center of mass system, and $\sigma(E)$ is the reaction cross section. As it was seen in the previous section, the term $\langle\sigma v\rangle$ increases by a factor

$$
E=\exp \left[-\frac{\Delta F}{k T}+\phi(\Gamma, \eta)\right]=\exp \left[\frac{\Delta \mu}{k T}+\phi(\Gamma, \eta)\right]
$$

but since the energy threshold must include the contribution of the Coulomb interactions to the chemical,potential: $Q=Q_{0}+\Delta \mu$, the photodesintegra tion rate is reduced by a factor $\exp (-\Delta \mu / k T)$ which cancels the dominant term of the screening factor (Mochkovitch, 1983; Mochkovitch and Nomoto, 1986). Therefore, the reaction rate becomes:

$$
\lambda=\lambda(\Gamma=0) e^{\phi(\Gamma, \eta)}
$$

and since $\phi(\Gamma, \eta)$ is negative, the photodesintegration rate is reduced instead of enhanced. 


\subsubsection{Electron polarization}

In all the previous calculations it has been assumed that the degenerate electrons form a uniform, perfectly rigid background. This approximation is justified since $\lambda_{T F} / a_{e}$, the ratio of the Thomas-Fermi screening length to the radius of the electronic sphere is larger than one in the regions of interest. Nevertheless, the polarization of electrons is not zero and this effect must be taken into account.

If the polarization of electrons is small, the Coulomb excess of free energy per ion, or the chemical potential, can be written in the form of a term computed assuming a uniform electron background plus a small correction due to the electron polarization (Mochkovitch and Hernanz 1986; Yakovlev and Shalybkov 1988): $\ln E=H_{i}+H_{p o l}$ where $H_{p o l}=-\Delta F_{p o l} / k T$ and $\Delta F_{p o l} / k T$ is the difference of polarization energies of electrons. Notice that despite this term is usually called electron screening, it is not the screening due to electrons but the perturbation induced by the polarization of electrons to the screening computed assuming a uniform electron background.

The contribution of electron polarization can be computed in the following way (Mochkovitch and Hernanz 1986):

$$
H_{p o l}=\frac{2}{\pi} q_{T F}^{2} \Gamma\left[2^{4 / 3} I\left(2^{5 / 3} \Gamma, x\right)-I(\Gamma, x)\right]
$$

where

$$
I(\Gamma, x)=\int_{0}^{\infty} \frac{S_{0}(q) F(x, y)}{q^{2}} d q
$$

$\mathrm{S}_{0}(\mathrm{q})$ is the structure factor corresponding to the pure Coulomb unperturbed potential, $F(x, y)$ is related to the dielectric function of relativistic electrons and $x=\hbar k_{F} / m_{e} c, y=q / q_{F}, q_{F}=(9 \pi Z / 4)^{1 / 3}, q_{T F}=a / \lambda_{T F}$, $q=a k$. In the case of a pure carbon plasma, $\mathrm{Z}=6, \rho=10^{9} \mathrm{~g} / \mathrm{cm}^{3}$ and $\mathrm{T}=10^{8} \mathrm{~K}, \mathrm{H}_{\text {pol }}=0.55$ while $\mathrm{H}_{i}=37.2$. Therefore, due to the uncertainties involved in the nuclear reactions and in the screening factor itself, it is usually possible to neglect the contribution of this term.

These results, however, strongly differ from those of Ichimaru (1993) who found enhancements much more important, by one or two orders of magnitude. This issue is very important and must be clearly elucidated (see Rosenfeld, this volume) since these corrections can completely modify the results discussed below about the accretion induced collapse of white dwarfs. Recent independent calculations by Sahrling (1994) confirm the results of Mochkovitch and Hernanz, and Yakovlev and Shalybkov. The effect of the electron polarisability in the afore-mentioned example is found to be negligible (of the order of $1 \%$ ). 


\subsubsection{Pycnonuclear reactions}

The evaluation of the reaction rate in the pycnonuclear regime is quite complicated since the energy distribution of nuclei is no longer given by the Boltzmann distribution and it is necessary to solve the Sch rödinger equation to obtain the wave function. The first discussion of this process was given by Cameron (1959) and was later elaborated by Wolf (1965), Salpeter and Van Horn (1969), Schramm and Koonin (1990) and Ogata et al (1991), in the case of one component plasma. The case of binary ionic mixtures has been recently treated by Ichimaru et al (1992), while the case of the $3 \alpha$ process has been treated by Salpeter and Van Horn (1969), Fushiki and Lamb (1987), Schramm et al (1992) and Langanke and Müller 1993).

\subsubsection{Fusion rates in one component plasma}

In the pycnonuclear regime, no independent particle model provides an adequate description of the fusion process and there are not simple approximations to the potential of the interacting particles. It is possible, however, to find two limiting cases for the potential (Salpeter and Van Horn, 1969): The "static" and the "relaxed" approximations. The first one assumes that all the nuclei as well as the center of masses of the reacting pair are frozen at the equilibrium positions, while the second one assumes that the position of the center of masses is fixed and the remaining lattice points polarize into the positions determined by the separation of the two reacting nuclei. Wolf (1965), using a simplified study of the dynamics of the crystal, obtained an intermediate potential between these two limiting cases, but nearer to the static case. Lately, Ogata et al (1991) determined the interparticle potential using a MonteCarlo method and found a result that is near to the relaxed approximation.

The result of Ogata et al (1991) can be easily understood in the following terms: Let $\Delta t$ be the time spent by a reacting pair with a typical energy $\hbar \omega_{p}$ into the barrier and let $\omega_{p}^{-1}$ be the characteristic response time of the crystal. Since $\omega_{p} \Delta t \simeq 140 Z^{5 / 2} \rho_{6}^{-1 / 4}$ (Schramm and Koonin 1991) is bigger than one for all the situations of physical interest, the lattice will respond adiabatically and will polarize under the influence of the reacting pair. Nevertheless, since the kinetic energy associated to the polarization must be taken into account during the calculation of the tunneling probability, it turns out that both effects of the polarization almost cancel and the final fusion rate is very near to the static one (Schramm and Koonin, 1991). The inclusion of dynamic terms has been contested by Ichimaru (1993) who argued that all the dynamic effects are already included in the 


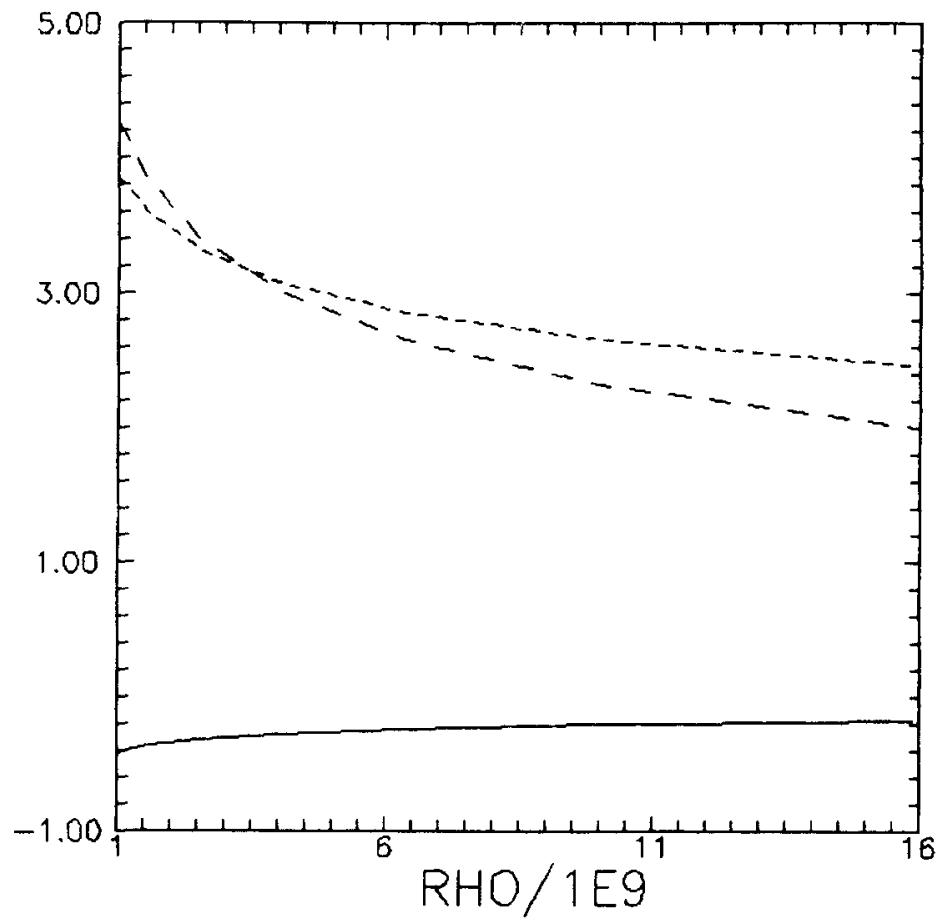

Fig. 5.3 Comparison of different fusion rates normalized to the static rates, $\log \left(R / R_{\text {static }}\right)$, versus $\lambda$, where $\lambda$ is the ratio of the nuclear Bohr radius and the lattice spacing $\left(\lambda \propto \rho^{1 / 3}\right)$. Solid line is from Schramm and Koonin (1991). Dashed line is from Ogata et al (1993). Dotted line corresponds to the relaxed approximation of Salpeter and Van Horn (1969).

pair correlation function through the dynamic structure factors since nuclear reactions are very rare events as compared with all the other collision processes. Figure 3 displays the comparison between the different fusion rates quoted here.

\subsubsection{Fusion rates in a binary ionic mixture}

In the case of a plasma with several components the situation is rather complicated because of the uncertainties about the structure of the crystal. Usually, the fusion rate has been taken as $\epsilon(x)=\epsilon(x=1) x^{2}$, where $\mathrm{x}$ is the molar fraction. The validity of this expression relies on the assumption that the reacting nuclei are randomly distributed and that the lattice potential is uniform.

The existence of lattice imperfections can be handled in the following way 
(Salpeter and Van Horn 1969): The presence of a default produces a local increase of the density that translates into an increase of the local rate by a factor $\exp \left(1.3 \lambda^{-1 / 2} \delta \lambda / \lambda\right)$, where $\delta \lambda$ is the local excess of the parameter $\lambda$. Because of the extreme sensitivity of the pycnonuclear reactions to the density, the nuclear fusion will start at these defaults and, if the relaxation of the reaction products produces new defaults, the reaction rate will be strongly enhanced.

The presence of other chemical species can have two different consequences. If the reacting and non-reacting nuclei have similar charges (case of $\mathrm{C}-\mathrm{O}$ mixtures) the heavier species blocks the reaction and its rate is strongly reduced. If they have very different values, the heaviest one induces a local increase of the density and the reaction rate is strongly enhanced. The critical value that separates both behaviors is $Z_{2} / Z_{1}=2.3$, where $Z_{2}>Z_{1}$. Figure 4 shows the influence the presence of oxygen and iron on the pycnonuclear fusion of carbon.

\subsubsection{The $3 \alpha$ fusion rate}

The $3 \alpha$ reaction in the pycnonuclear regime was considered for the first time by Cameron (1959) and later on by Salpeter and Van Horn (1969), Fushiki and Lamb (1987) and Schramm et al (1992). Besides the problem of its three-body nature, the pycnonuclear $3 \alpha$ reaction is characterized by the fact that temperatures are too low to allow the resonance of ${ }^{8} \mathrm{Be}$ and by the fact that in the region of interest helium is a quantum fluid rather than a Coulomb lattice. The present results are quite satisfactory since different approaches give the same results. Nevertheless, the effect of the presence of impurities remains to be studied. It is interesting to notice that above $\rho \simeq 2-310^{9} \mathrm{~g} / \mathrm{cm}^{3}$ a lattice made of ${ }^{8} \mathrm{Be}$ has a lower energy than a ${ }^{4}$ He fluid (Schramm et al 1992) and ${ }^{8} \mathrm{Be}$ becomes stable. However, the pycnonuclear $3 \alpha$ reaction is able to convert helium into carbon before reaching this density and the possibility of the existence of ${ }^{8} \mathrm{Be}$ matter seems to be prevented (Langanke and Müller, 1993).

\subsection{The astrophysical problem}

\subsubsection{Collapse or explosion of mass accreting white dwarfs}

More than fifteen years ago, Schatzman suggested that besides the standard mechanism for neutron star formation (collapse of the fuel exhausted core of a massive star), a second mechanism was possible: gravitational collapse of a white dwarf due to accretion of matter in a close binary system. In 


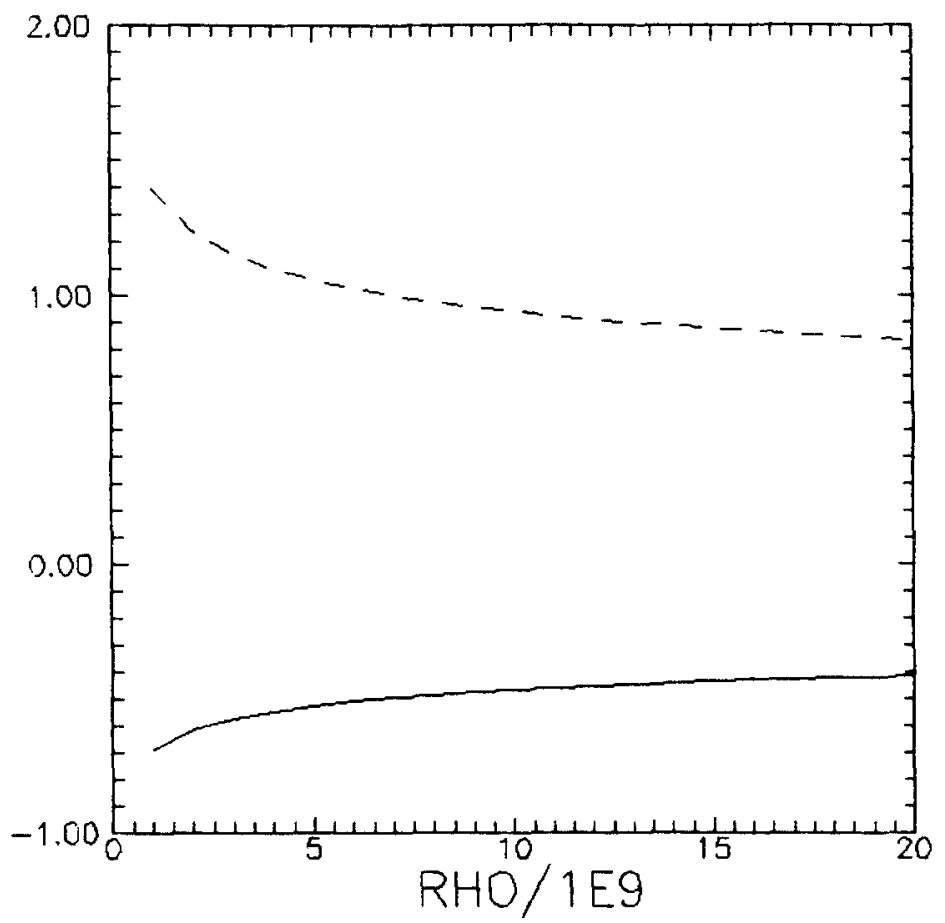

Fig. 5.4 Blocking and catalyzing effects of impurities in the pycnonuclear fusion of carbon. The vertical axis displays $\log [R(x \neq 0) / R(x=0)]$ for the ${ }^{12} \mathrm{C}+{ }^{12} \mathrm{C}$ reaction in the presence of oxygen (continuous line) and iron (dashed line). In both cases the molar abundance of the heavier species is $x=0.5$

both cases, collapse would be induced by growth above the effective Chandrasekhar's mass. The second mechanism would account for the presence of neutron stars in systems such as the low-mass X-ray binaries, where survival to a Type I supernova explosion appears unlikely. Accretion induced collapse of white dwarfs has later been incorporated in evolutionary scenarios for the origin of several kinds of binary systems containing neutron stars (Van den Heuvel 1983, 1989).

Even if white dwarfs might grow in mass at any arbitrary rate, which is not the case (Isern et al 1983; Hernanz et al 1988; Canal et al 1990), accretion induced collapse would in any case encounter the difficulty that explosive ignition of the nuclear fuel in the electron degenerate cores always precedes reaching the Chandrasekhar's mass. For white dwarfs made of carbon-oxygen or oxygen-neon-magnesium, ignition can be delayed until losses due to electron captures overcome the energy released by ther- 
monuclear reactions (Isern and Canal, this volume). CO white dwarfs were proposed by Canal and Schatzman (1976) while ONeMg white dwarfs were advocated by Miyaji et al (1980). Concerning CO white dwarfs, it was soon realized that central ignition in a partially solid core provided the most favorable conditions for accretion induced collapse (Canal and Schatzman 1976; Canal and Isern 1979).

The behavior of a cool white dwarf interior during the accretion phase depends on the competition between physical processes that increase the temperature of the material (compression, nuclear reactions in the inner core and thermonuclear burning of the accreted matter) and those that cool down the star (photon and neutrino losses). Obviously, heat conduction plays an important role, as being the main mechanism for heat transport in the stellar interior. Conduction can be characterized by the time required for a thermal signal to travel from the center to the surface of the star. This time is given by Henyey and L'Ecuyer (1969) as:

$$
\tau_{t h}=\frac{3 \kappa \rho^{2} c_{p}}{64 \sigma T^{3}} l^{2}
$$

where $l$ is the linear extent of the region considered and all the remaining symbols have their usual meanings. The time scale for increasing central density, when mass approaches the Chandrasekhar's limit, can be expressed as (Canal and Schatzman 1976):

$$
\tau_{\rho}=\left(\frac{M}{\dot{M}}\right)\left(\frac{10.08}{y_{0}^{3}}-8.310^{-4}\right) \frac{y_{0}}{3}
$$

where $\dot{M}$ is the mass accretion rate and $y_{0}=\sqrt{1+x_{F}^{2}}$, where $\mathrm{x}_{F}$ is the dimensionless Fermi momentum.

The effects of the compression induced by the accreted matter can be divided in two terms (Nomoto 1982). The first one is due to the increase in density at a fixed mass fraction as the stellar mass increases, and its effects are quite uniform throughout the whole star. The second term corresponds to compression as matter moves inwards in the mass-fraction space. It is negligible in the inner strongly degenerate regions of the star, but it is very large in the semi degenerate external layers. This means that a thermal wave is generated in those layers which diffuses inwards. A rough estimate of the compressional luminosity of the external layers is (Nomoto 1982):

$$
\frac{L_{g}^{N H}}{L_{\odot}}=1.410^{-3} T_{7} \dot{M}_{10}
$$


$\mathrm{T}_{7}$ being the temperature in units of $10^{7} \mathrm{~K}$ and $\dot{M}_{10}$ the accretion rate in units of $10^{-10} \mathrm{M}_{\odot} / \mathrm{yr}$.

The effects of this thermal wave on the temperature profile, and thus on the physical state of the white dwarf interior, will depend on the time needed for the thermal wave to reach the center of the star as compared to the time required for the star to reach the Chandrasekhar's limit and also on the efficiency of thermal cooling of the white dwarf (Hernanz et al 1988).

For low accretion rates $\left(10^{-12} \mathrm{M}_{\odot} / \mathrm{yr} \leq \dot{M} \leq 310^{-10} \mathrm{M}_{\odot} / \mathrm{yr}\right)$, the thermal wave generated by the accretion process in the external layers can reach the center of the star before any instability starts, but compression is so slow that radiative cooling through the star surface dominates. The white dwarf, in this case, evolves keeping an isothermal profile and the internal temperature is determined by the balance between compression and cooling through the envelope.

For high accretion rates $\left(\dot{M} \geq 510^{-8} \mathrm{M}_{\odot} / \mathrm{yr}\right)$, compressional luminosity is much higher. However, in this case, the thermal wave has no time to reach the center for high enough masses, and only the outermost solid layers are affected. In the central solid layers $\tau_{T h}>>\tau_{\rho}$ and they evolve quasiadiabatically. The slope for the evolutionary path in the $\log \rho-\log T$ diagram is given by (Mochkovitch 1983)

$$
\Gamma_{3}-1=\frac{0.815+0.215 \Gamma^{1 / 4}}{0.945+0.646 \Gamma^{1 / 4}}
$$

$\Gamma_{3}-1$ being the adiabatic index. For $\Gamma$ values in the range $100-200$, the adiabatic index is $\sim 0.5$,

For intermediate accretion rates, $\left(310^{-10} \mathrm{M}_{\odot} / \mathrm{yr} \leq \dot{M} \leq 510^{-8} \mathrm{M}_{\odot} / \mathrm{yr}\right)$, the thermal wave has again enough time to reach the center of the star. Compressional heating dominates the normal cooling and matter heats up.

The outcome, collapse or explosion, depends on the density at which the thermonuclear runaway starts and this critical density depends on the velocity at which the burning front propagates. The minimum density necessary to get a collapse is $8.510^{9} \mathrm{~g} / \mathrm{cm}^{3}$ (Isern and Canal, this volume). Garcia and Bravo, also this volume, propose an even smaller value, $710^{9} \mathrm{~g} / \mathrm{cm}^{3}$. However, if all the modes of propagation of the burning front are taken into account, this critical density adopts a value in the range 9.2 to $9.510^{9}$ $\mathrm{g} / \mathrm{cm}^{3}$, although these limits are rather uncertain.

It is thus interesting to examine the dependence of the ignition density on the adopted value of the nuclear reaction rate. Table 1 displays the ignition density for different accretion rates. All the models have been constructed in the same way as in Hernanz et al (1988). Models labeled with a capital letter 
Table 5.1. Ignition densities (in units of $10^{9} \mathrm{~g} / \mathrm{cm}^{3}$ ) for different pycnonuclear reaction rates

\begin{tabular}{llllll}
\hline \hline$\dot{M}\left(M_{\odot} / y r\right)$ & $\mathrm{A}$ & $\mathrm{B}$ & $\mathrm{a}$ & $\mathrm{b}$ & $\mathrm{c}$ \\
\hline $10^{-6}$ & 10.60 & 12.40 & 10.40 & 12.10 & 10.85 \\
$10^{-7}$ & 9.05 & 10.10 & 6.63 & 6.63 & 6.51 \\
$10^{-9}$ & 7.87 & 9.10 & 7.82 & 8.97 & 7.99 \\
$10^{-10}$ & 7.98 & 9.92 & 7.98 & 9.89 & 8.26 \\
$10^{-11}$ & 7.73 & 9.66 & 7.71 & 9.63 & \\
\hline \hline
\end{tabular}

started at $M_{W D}^{0}=1.2 M_{\odot}$, those with a small letter started at 1.1. The initial temperature was $T_{0}=410^{6} \mathrm{~K}$ for all of them. Pycnonuclear reaction rates from Ogata et al (1991) were used in models A and a. Models B and b were obtained using the rates of Schramm and Koonin (1990), while model c was obtained with the pycnonuclear rates of Ichimaru et al (1992), which include the blocking effects due to the presence of oxygen nuclei.

Columns $A$ and $B$ show the importance of including the dynamics of the crystal in the reaction rates. In the first case, almost all of them collapse. Comparison of model $c$ with a shows that the blocking effects of oxygen effectively delay the runaway but do not introduce any qualitative change in the above picture.

The initial mass is also a critical factor. Below $1.05 \mathrm{M}_{\odot}$, the accretion induced collapse is impossible. Since the outer thermal wave has less time to reach the centre, collapse is favored by increasing the initial mass. This implies that only the tail of most massive $\mathrm{CO}$ white dwarf can collapse to form a neutron star.

The strong screening factors adopted and the interpolation algorithm be tween strong screening and pycnonuclear regimes are also critical. Table 2 displays the ignition density in the case of a white dwarf with an initial mass $\mathrm{M}_{W D}=1.2 \mathrm{M}_{\odot}$, initial temperature $\mathrm{T}=410^{6} \mathrm{~K}$, which accretes matter at a rate $\dot{M}=10^{-7} M_{\odot} / y r$ and is made of carbon and oxygen. The screening factors have been obtained from Ogata et al (1991), OII model, and from Rosenfeld (1993) and Alastuey and Jancovici (1979), RAJ model. The pycnonuclear rate was obtained from Ichimaru et al (1993). The gap between pycnonuclear and strong screening reactions was covered with the interpolation algorithm described by Bravo et al (1983). In model $A$, the rates were obtained interpolating between the line $3 \Gamma / \tau=1$ (strong screening regime) and $3 \Gamma / \tau=8.1$ (pycnonuclear region), Model $\mathrm{B}$ by interpolating between $3 \Gamma / \tau=1$ and $\Gamma=180$ and model $C$ between $3 \Gamma / \tau=1.5$ and $\Gamma=180$. As it 
Table 5.2. Ignition densities (in units of $10^{9} \mathrm{~g} / \mathrm{cm}^{3}$ ) for different strong screening factors

\begin{tabular}{llll}
\hline \hline & A & B & C \\
\hline OII & 9.23 & 9.90 & off \\
RAJ & 8.88 & 9.70 & 9.88 \\
\hline \hline
\end{tabular}

can be seen from table 2, the results are spectacular and even an off center ignition is obtained in case $\mathrm{C}$ model OII.

\subsubsection{Slowly accreting neutron stars}

Neutron stars are rather abundant in the Galaxy. Their estimated density in the solar neighborhood is $n \simeq 10^{4} /(300 p c)^{3}$. Few of them can be detected as pulsars, if they are young enough or/and they rotate fast enough, or as bright $\mathrm{X}$-ray sources, if they are members of a close binary system (in this case, they accrete matter at a rate $\dot{M} \simeq 10^{-8} M_{\odot} / y r$ and they emit $L_{X} \simeq 10^{38} \mathrm{erg} / \mathrm{s}$ in $\mathrm{X}$-rays). The question is: what happens with the remaining, isolated and old, neutron stars that accrete at a typical rate of $10^{10} \mathrm{~g} / \mathrm{s}$ ?.

The evolution of neutron stars that accrete mass at a very low rate, $10^{-16} \leq \dot{M} \leq 10^{-11} M_{\odot} / y r$, has been studied many times (Hameury et al 1983; Blaes et al 1989, 1990, 1992; Miralda-Escudé et al 1990; Zdunik et al 1992) In all of them the pycnonuclear rates have proved to play a critical role since the mass-accretion is so slow that the temperature is too low to allow thermonuclear rearrangement of nuclei. Their evolution can be summarized in the following way:

a) Few years after the explosion, the neutron star captures the bound debris and burns them to ${ }^{56} \mathrm{Fe}$. Because of the weight of the newly accreted matter, this material sinks and starts capturing electrons: ${ }^{56} \mathrm{Fe} \rightarrow{ }^{56} \mathrm{Cr} \rightarrow{ }^{56} \mathrm{Ti}$.

b) After the pulsar phase, the neutron star can start accreting matter from the interstellar medium, $\dot{M} \simeq 10^{10} v_{40}^{-3} n_{I S M} \mathrm{~g} / \mathrm{s}$, where $v_{40}$ is the speed of the star relative to the interstellar medium in units of $40 \mathrm{~km} / \mathrm{s}$ and $n_{I S M}$ is the hydrogen number density of the interstellar gas in $\mathrm{cm}^{-3}$ (Hoyle and Lyttleton, 1939), which imply accretion rates of the order of $10^{10} \mathrm{~g} / \mathrm{s}$. The newly accreted matter compresses the crust and hydrogen is burnt to helium due to pycnonuclear reactions or electron captures, depending on the accretion rate. Since spallation reactions at the surface of the star destroy most of the metals (Bildstein et al 1991), burning is stable as far 
as $\dot{M} \leq 210^{13} \mathrm{~g} / \mathrm{s}$ and the crust can be considered isothermal, $T_{6}=\dot{M}_{10}^{5 / 11}$ (Blaes et al 1992, for such low rates.

c) The helium newly formed in this way solidifies at a temperature $T_{s} \simeq$ $3.310^{3} \rho^{1 / 3}$. For an isothermal crust of the kind mentioned in $b$ ), this will happen at a density $\rho_{s}=2.810^{7} \dot{M}_{10}^{15 / 11}$ (Blaes et al 1992). As He behaves like a quantum liquid for densities higher than $310^{8} \mathrm{~g} / \mathrm{cm}^{3}$, (Mochkovitch and Hansen 1979, Chabrier 1993), a layer of solid He surrounded by a classical liquid in the top and a quantum liquid in the bottom will form. When the density is high enough, helium ignites due to pycnonuclear reactions. The characteristic time scales for the $\alpha$-captures are:

$$
\begin{gathered}
\left.\tau\left[{ }^{12} C(\alpha, \gamma)\right)^{16} O\right] \simeq 10^{-9} s \\
\tau\left[{ }^{16} O(\alpha, \gamma)^{20} N e\right] \simeq 10^{15} s
\end{gathered}
$$

Thus, if the $3 \alpha$-reaction were slow, the freshly synthesized oxygen would have time to capture an $\alpha$-particle and approach to $\mathrm{Si}$. Since the $3 \alpha$ rate is very high (Schramm, Langanke and Koonin, 1991) only ${ }^{12} \mathrm{C}$ has time to capture an $\alpha$-particle and an ${ }^{16} \mathrm{O}$-rich mixture forms. As oxygen crystallizes at higher temperatures than helium $\left(\rho_{c} \simeq 1.310^{5} \dot{M}_{10}^{15 / 11} \mathrm{~g} / \mathrm{cm}^{3}\right)$, it immediately solidifies and sinks, accumulating at the bottom of the quantum He-ocean.

d) As the accretion proceeds, the density at the bottom of the accreted material increases and finally at $\rho=1.9210^{10} \mathrm{~g} / \mathrm{cm}^{3},{ }^{16} \mathrm{O}$ undergoes a twostage electron capture to ${ }^{16} \mathrm{C}$. If this ${ }^{16} \mathrm{C}$ accumulates or fuses depends on the pycnonuclear rate of the reaction ${ }^{16} C+{ }^{16} C$. If it accumulates, it can undergo an elastic Rayleigh-Taylor instability with the old underlying crust resulting in a starquake which releases some $10^{40}$ ergs of gravitational and nuclear energy (Blaes et al 1990, 1992).

The fusion of ${ }^{16} \mathrm{C}$ was studied by Bravo et al (1983). It has the following characteristics:

$$
\begin{aligned}
& { }^{16} \mathrm{C}+{ }^{16} \mathrm{C} \rightarrow{ }^{28} \mathrm{Mg}+4 n(90 \%) Q=10.1 \mathrm{MeV} \\
& { }^{16} \mathrm{C}+{ }^{16} \mathrm{C} \rightarrow{ }^{29} \mathrm{Mg}+3 n(10 \%) Q=14.6 \mathrm{MeV}
\end{aligned}
$$

with $S_{0}=5.510^{8} \mathrm{MeV}$-barn. At the density at which it is produced, its characteristic fusion time scale is $1000 \mathrm{yrs}$, quantity that can be much shorter if some heavy elements coming from the original crust diffuse and mix with the ${ }^{16} \mathrm{C}$ layers. Therefore, the composition will change to ${ }^{28} \mathrm{Mg}$. During all this process, the underlying original crust makes a transition to 
${ }^{56} \mathrm{Ti},{ }^{62} \mathrm{Cr}$ or ${ }^{62} \mathrm{Fe}$ because of electron captures and, since its mean molecular weight per electron is equal or higher than that corresponding to ${ }^{28} \mathrm{Mg}$, the accreted layer will be stable.

\subsection{Conclusions}

Carbon-oxygen white dwarfs can either collapse or explode depending on their initial properties. If they are massive and cool enough, they can nonexplosively collapse and form a neutron star. The frequency and relevance of this phenomenon, as compared with the accretion induced collapse of ONeMg white dwarfs, critically depends on the adopted rates and on the way as the transition between strong screening and pycnonuclear regimes is handled. The effects due to the polarization of electrons can be critical. If they increase the reaction rates by one or two orders of magnitude as claimed by Ichimaru (1983), the collapse to a neutron star will be prevented in the majority of cases.

The possibility of observing isolated neutron not detectable as pulsars also depends on the adopted pycnonuclear rates. If the rates are high enough, due to the presence of defaults or heavy impurities, the slowly accreted material is gradually transformed and neutronized without forming a metastable layer that could release its energy in a burst.

Therefore, due to the current uncertainties on the screening factors it is not possible to reach a definite conclusion in both cases. It should be of the highest interest to definitively elucidate the behavior of nuclear reaction rates at high densities $(3 \Gamma / \tau>1)$ and in the pycnonuclear region $(\Gamma>180)$.

Acknowledgements This work has been partially financed by the DGICYT grant PB91-060, the Spanish-French Action "Physics of White Dwarfs and Brown dwarfs" and the CESCA grants "Structure and Evolution of Galaxies" and "Accretion onto White Dwarfs".

\section{References}

Alastuey A., Jancovici B., Astrophys. J. 226, 1034 (1978)

Bravo E., Isern J., Canal R., Labay J., Astron. Astrophys.124, 39 (1983)

Bildsten L., Salpeter E.E., Wasserman I.,Astroph. J.384, 143 (1992)

Blaes O., Blandford R., Goldreich P. and Madan P.,Astrophys. J.343, 839 (1989)

Blaes O., Blandford R., Madan P. and Koonin S.,Astrophys. J363, 612 (1990)

Blaes O., Blandford R., Madan P. and Yan L., Astrophys. J.399, 634 (1992)

Cameron A.G.W., Astrophys. J. 130, 916 (1959)

Canal R., Isern., in White Dwarfs and Variable Degenerate Stars IAU Colloq

No153, ed H.M. Van Horn, V. Weidemann, p.52, Rochester NY: Univ.

Rochester Press (1979) 
Canal R., Isern J., Labay J., Ann. Rev. Astron. Astrophys. 28, 183 (1990)

Canal R., Schatzman E., Astron. Astrophys. 46, 229 (1976)

Chabrier G., Astrophys. J. 414, 695 (1993)

DeWitt H.E., Graboske H.C., Cooper M.S.,Astrophys. J. 181, 439 (1973)

Graboske H.C., DeWitt H.E., Grossman A.S., Cooper M.S.,Astrophys. J. 181, 457 (1973)

Fushiki I., Lamb D.Q., Astrophys. J. 317, 368 (1987)

Hameury J.M., Heyvaerts J. and Bonazzola S., Astron. Astrophys.121, 259 (1983)

Helfer H.L., McCrory R., Van Horn H,M., J. Stat. Phys 37, 577 (1984)

Henyey L., L'Ecuyer J ., Astrophys. J. 156, 549 (1969)

Hernanz M., Isern J., Canal R., Labay J., Mochkovitch R., Astrophys. J. 324, 331 (1988)

Hoyle F. and Lyttleton R.A.,Proc. Cambridge Phil. Soc.35, 592 (1939)

Ichimaru S., Rev. Mod. Phys. 65, 255 (1973)

Ichimaru S., Ogata S., Van Horn H.M., Astrophys. J. Letters 401, L35 (1992)

Isern J., Labay J., Hernanz M., Canal R., Astrophys. J. 273, 320 (1983)

Itoh N., Kuwashima F., Munakata H.,Astrophys. J. 362, 620 (1990)

Itoh N., Totsuji H., Ichimaru S., Astrophys. J. 220, 742 (1978)

Itoh N., Totsuji H., Ichimaru S., DeWitt H.E.,Astrophys. J. 1079, (1979)

Jancovici B., J. Stat. Phys. 17, 357 (1977)

Miralda-Escudé J., Haensel P. and Paczynski B.,Astrophys. J.362, 572 (1990)

Miyaji S., Nomoto K., Yokoi K., Sugimoto D., Publ. Astron. soc. Japan 32, 303 (1980)

Mochkovitch R., Astron. Astrophys. 122, 212 (1983)

Mochkovitch R., Hansen J.P.,Phys. lett.73A, 35 (1979)

Mochkovitch R., Hernanz, M., in Nucleosynthesis and its implications on nuclear and particle physics, eds. J. Audouze and J. Trân Thanh Vân, p.109, Reidel: Dordretch (1986)

Nomoto K., Astrophys. J. 257, 780 (1982)

Ogata S., Ichimaru S., Phys. Rev A36, 5451 (1987)

Ogata S., Iyetomi H., Ichimaru S., Astrophys. J. 372, 259 (1991)

Pines D, Nozières P., The theory of quantum liquids (Benjamin: New York) (1966)

Sahrling M., to appear in $A \mathscr{E} A$, (1994)

Salpeter E.E., Aust. J. Phys. 7, 373 (1954)

Salpeter E.E., Van Horn H.M., Astrophys. J 155, 183 (1969)

Schatzman E., presented at InT. Sch. Cosmol. Gravit. Erice, Italy (1974)

Schramm S., Koonin S.E., Astrophys. J. 365, 296 (1990)

Schramm S., Langanke K and Koonin S.E.,Astrophys. J.397, 579 (1992)

Slattery W.L., Doolen G.D., DeWitt H.E., Phys Rev A21, 2087 (1980)

Slattery W.L., Doolen G.D., DeWitt H.E., Phys Rev A26, 2355 (1982)

Van den Heuvel E.P.J., in Accretion-Driven Stellar X-Ray Sources Ed. W.H.G. Lewin, E.P.J. Van den Heuvel, p.303, Cambridge Univ. Press ()

Van den Heuvel E.P.J., in Timing Neutron Stars, ed H.O. Ogelman E.P.J. Van den Heuvel, Dordrecht: Kluwer, p.523 (1989)

Yakovlev D.G., Shalybkov D.A., Adv. Space. Res. 8, (2)707 (1988)

Zdunik J.L., Haensel P., Paczynski B. and Miralda-Escudé J., Astrophys. J.384, 129 (1992) 